\title{
SINUS TYMPANI: A PRACTICAL CLASSIFICATION
}

\author{
$\mathscr{B}_{4}$ \\ Alaa A. Abou-Bieh, MD1, Thomas J Haberkamp, MD² \\ From \\ ${ }^{1}$ Department of Otolaryngology, Faculty of Medicine, \\ Mansoura University, Mansoura, Egypt \\ ${ }^{2}$ Cleveland Clinic, Cleveland, OH, USA
}

\begin{abstract}
objectives:

1- Describe a classification for the sinus tympani (ST) different types based on their otoendoscopic and surgical anatomy in relation to their development theories.

2- Evaluate possible approaches to each of its types.

3- Eliminate the discrepancy between the various methods used to describe it in literature.

\section{Methods:}

Fifty-five temporal bones were dissected, and the anatomical details were studied utilizing an operating microscope and otoendoscopes of different angles. In addition, the ST anatomy and relations were studied in two hundred temporal bones CT scans.
\end{abstract}

\section{Results:}

Four distinct types of ST could be observed. Type 1, the most common type, it was pneumatized and consisted of an orifice and cavity. Type 2 , the well pneumatized type, the cavity was deep enough posteriorly to exceed the level of the mastoid segment of the facial nerve in any direction. Type 3 , the common posterior tympanic sinus, in which ST communicated with the upper posterior tympanic sinus proper or with a retro-ponticulus up-ward extension. Type 4, the non- pneumatized type, was shallow with no true orifice and cavity. Type 1 and 4 were mainly approached via the trans-canal route.

Type 2 and 3 required a combined trans-canal and trans-mastoid approaches. Endoscopic trans-canal MANSOURA MEDICAL JOURNAL 
approach alone was satisfactory for types 1 and 4 but to less extent type 3.

\section{Conclusions:}

ST shape and extension both depend mainly on the extent of its pneumatization, which in turn influences its relations to the surrounding structures in the retro-tympanic area. Extensively or unusually pneumatized types need special or combined approaches.

\section{INTRODUCTION}

The sinus tympani (ST) is one of the recesses of the posterior tympanum (retrotympanum) and it is one of the sinuses of the posterior tympanic wall which formed by the abutting of the primitive endothelial pouches of the eustachian tube origin as they develop the middle ear air system namely the saccus posticus-against the solid upper portion of the Reichert's cartilage of the second branchial arch which ossifies to develop the styloid complex of the Posterior tympanic wall. 1 Therefore it lies between the labyrinthine capsule medially, posteriorly (posterior semicircular canal) and superiorly (lateral semicircular canal), the sty- loid complex [the pyramidal eminence(PE), the styloid eminence (SE) and the chordal eminence (CE)] laterally and the facial nerve (the nerve of the second arch) with its bony canal posteriorly, posterolaterally or laterally. 1,2 Inferiorly, ST is limited by the bony ridge between the styloid eminence and bony labyrinth the subiculum (S) and to variable degree, by the jugular wall,.1 $\mathrm{A}$ bony or membranous ridge or bridge the ponticulus (P) sometimes present between pyramid and promontory.1 Anatomical studies noted the large anatomic variations of the ST and tried to classify it according to variations in the size, extension and depth3,4,5,6,7 or shape8. The importance of ST comes from that it is often invaded by cholesteatoma and/or granulation tissue and also being well known for the high risk of residual and recurrent disease, because conventional surgical approaches always failed to provide adequate access to a deep sinus tympani to eradicate disease. 2 Some authors suggested different surgical approaches to the ST including the retrofacial 7,9 and the combined 10 approaches to be possible solutions of this problem. Although the otoen- 
Alaa A. Abou-Bieh, MD, Thomas J Haberkamp MD

doscopic surgery has improved visualization of this area in recent years, sometimes it does not solve the problem to remove the diseased tissue completely alone.11,12,13

The aim o甲 this study is to describe a classification for the ${ }^{\circledR S T}$ different types based on their otoendoscopic and surgical anatomy in relation to their developmen ${ }^{\oplus}$ theories. By combining these three elements, we aim to find a simple, practical and clinically significant classification and at the same time to eliminate the discrepancy between the various methods used to describe it in literature. The main significance of this classification would be evaluating possible approaches to each of its types in a way that makes it possible to decide the surgical approach preoperatively in most of cases. In addition, to find a simple effective fixed way of to describe and report cases.

\section{MATERIALS AND METHODS}

The gross anatomy and anatomical variations of posterior mesotympanum and retrotympanic areas including the ST were studied in 55 Fresh frozen temporal bones. To the best of our knowledge, all bones came from adult cadavers. There were 31 right temporal bones and 24 left. All bones were dissected by the same approach and all were studied utiliang an operating microscope and otoendoscopes with 00,300 and ongles and 2.7 and $3 \mathrm{~mm}$ diameters. The photos were processed digitally by Photoswit ${ }^{\circledR}$ digital computer photo-system (Photoswit, USA). Measurements were taken by the use of paparella Fenestrometer (Gyrus $\AA$, USA). In addition, the ST anatomy and relations were studied in two hundred temporal bones CT scans $(=400$ temporal bones $)$. These scans were randomly chosen regard less they were of normal individuals or showing any type of temporal bone pathology, but all were for adult personals.

\section{RESULTS}

Four distinct types of ST could be observed in both temporal bone dissection and CT scans studying:

Type 1, it was pneumatized and consisted of an orifice and cavity. The orifice was bounded superiorly by the ponticulus $(P)$, inferiorly by the subiculum (S), posteriorly by the 
pyramidal crest (PC) and anteriorly by the promontory edge. The cavity was bounded laterally by the facial nerve canal (FNC), medially by the labyrinthine capsule, posteriorly by Posterior (vertical) semicircular canal (PSCC), superiorly by the posterior tympanic sinus (PTS) and Lateral (horizontal) semicircular canal (LSCC) and inferiorly by the styloid eminence (SE), subiculum (S) and the jugular dome. The cavity did not exceed the level of the mastoid segment of the facial nerve dorsally at all. This was the most common type $(49.7 \%)$ presented in 24 temporal bones (43.6\%) and 202 ears in the CT scans $(50.5 \%)$. In the temporal bone study this type was easily approached trans-canal microscopically and endoscopically assisted.

Type 2, the well pneumatized type, it was formed from an orifice and cavity or an orifice, vestibule and cavity. The cavity was deep enough posteriorly to exceed the level of the mastoid segment of the facial nerve in any direction. Its percentage was $(31.6 \%)$ and occurred in 9 temporal bones (16.3\%) and 135 ears in the CT scans (33.8\%). In the temporal bone study this type neces- sitate to be approached via a transmastoid retro-facial approach or by a combined trans-canal (microscopically and endoscopically assisted) and trans-mastoid approach. It was usually associated with very well pneumatized mastoids.

Type 3, the common posterior tympanic sinus, in which ST communicated with the upper posterior tympanic sinus proper or with a retroponticulus up-ward extension. This category of ST should be subdivided into deep and very deep according to either the cavity would extend beyond the level of the mastoid segment of the facial nerve in any direction or not. It occurred in 20 temporal bones (36.3\%). It was possible to expect it in CT scans only rarely and accidentally so we did not include it in CT scan calculations. In the temporal bone study this type necessitate to be approached via a transmastoid approach or by a combined trans-canal (microscopically and endoscopically assisted) and transmastoid approach depending on the depth of its cavity same as types 1 and 2 .

Type 4, the non- pneumatized 
Alaa A. Abou-Bieh, MD, Thomas J Haberkamp MD

type, was shallow with no true orifice and cavity. The presence of the ponticulus $(P)$, the subiculum $(S)$ and the pyramidal crest $(P C)$ was variable and not consistent. Its percentage was $(14.3 \%)$ and occurred in 2 temporal bones $(3.6 \%)$ and 63 ears in the CT scans (15.8\%). Most of the CT scans showed this type were of unhealthy temporal bones. It was easily approached trans-canal.

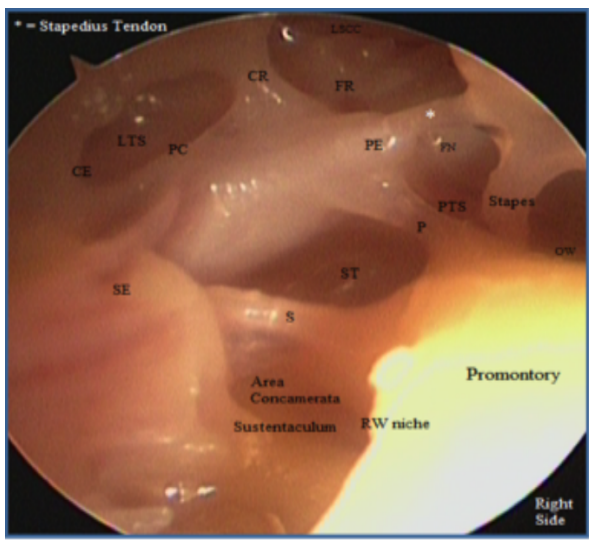

Figure 1. Type 1ST Endoscopic View
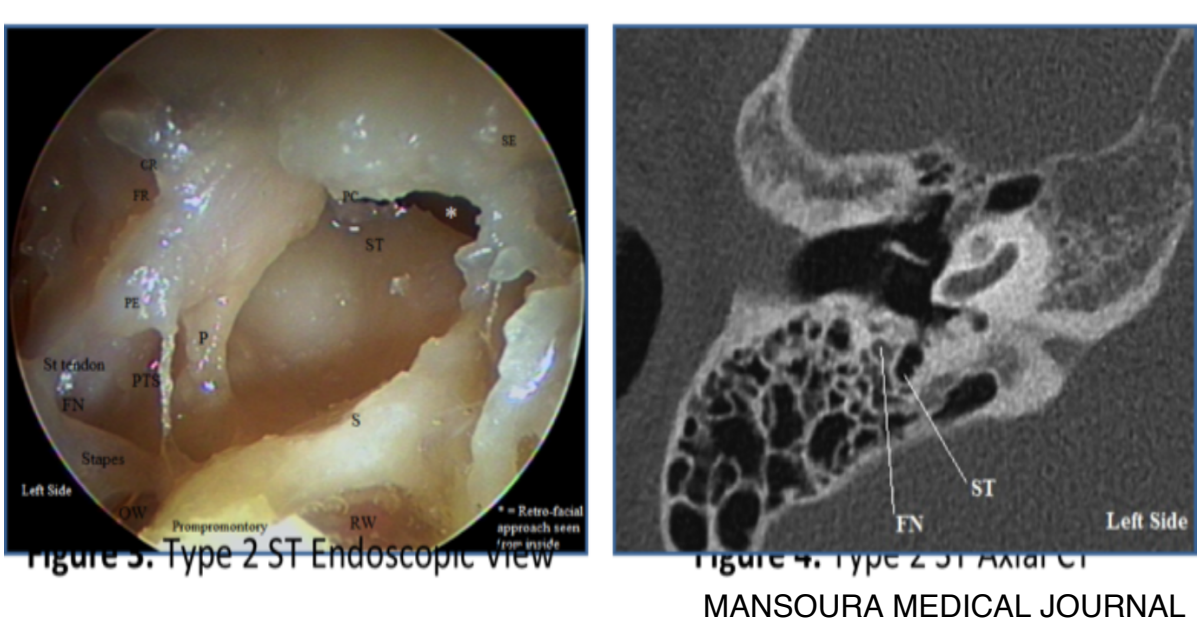

MANSOURA MEDICAL JOURNAL

In 79 ears in CT scans there was a difference between the ST type in one side than the other. Either one was shallow and the other was pneumatized or one was pneumatized and the other was well pneumatized. Endoscopic transcanal approach alone was satisfactory for types 1 and 4 but to less extent type 3.

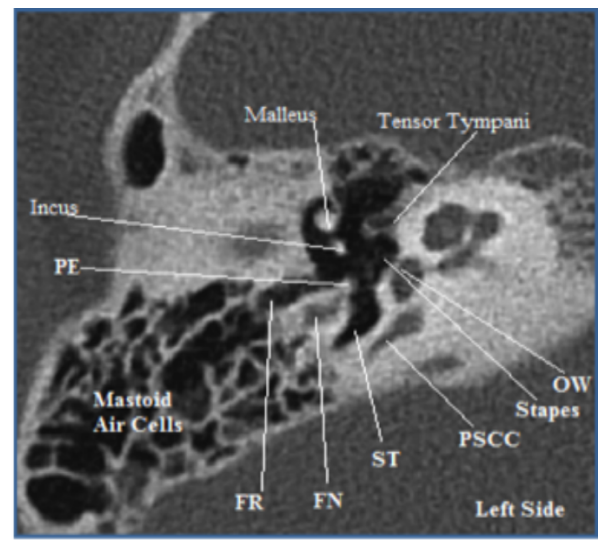

Figure 2. Type 1 ST Axial CT 


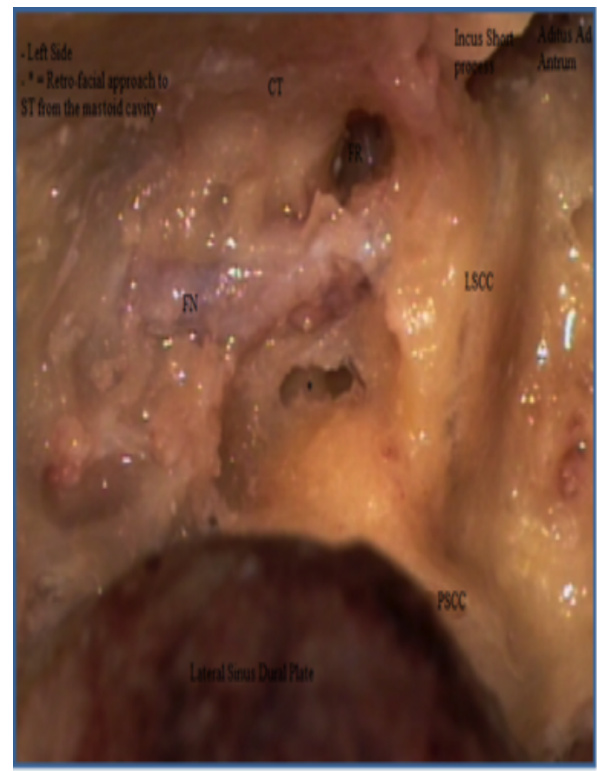

Figure 5. Type 2ST Retrofacial APP

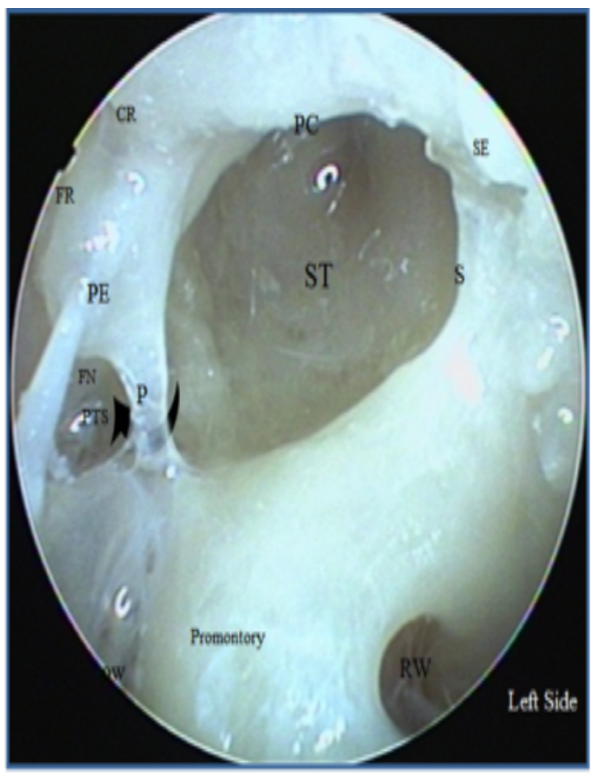

Figure 6. Type 3ST Endoscopic View

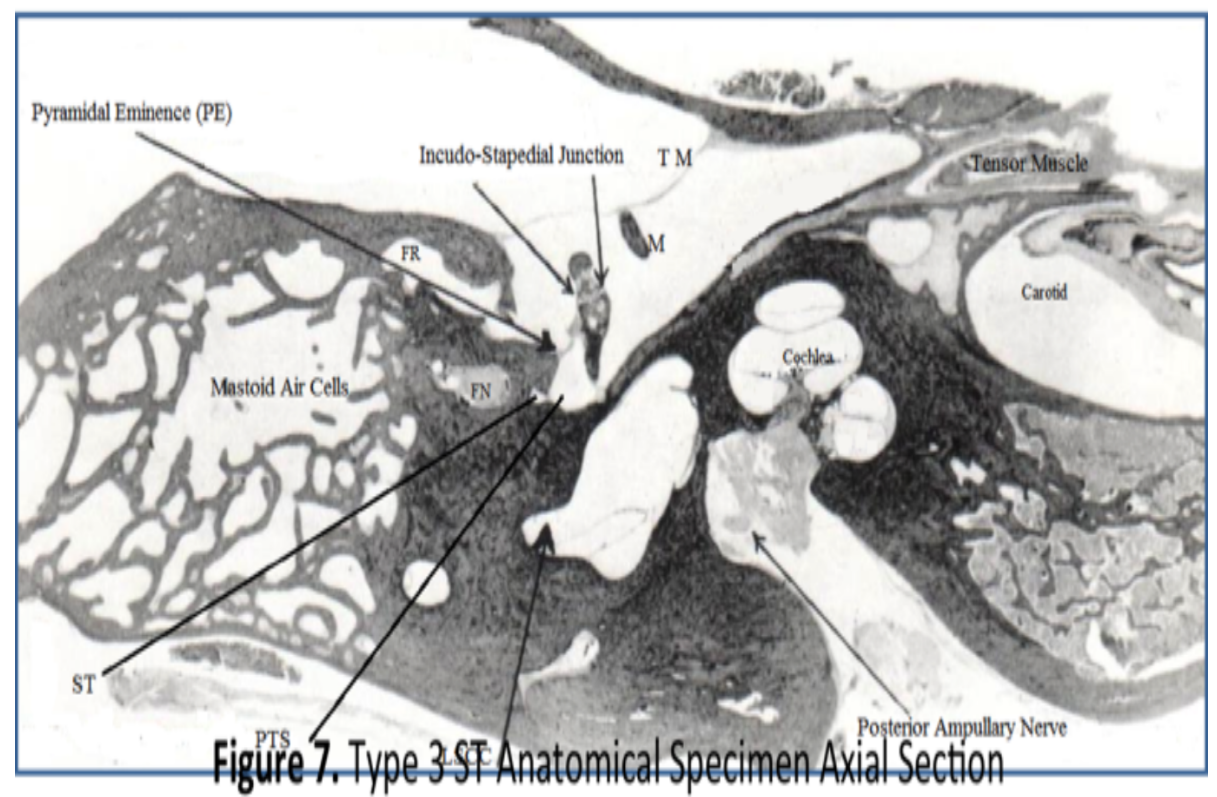

Vol. 44, No. 1 \& 2 Jan. \& April, 2015 
Alaa A. Abou-Bieh, MD, Thomas J Haberkamp MD

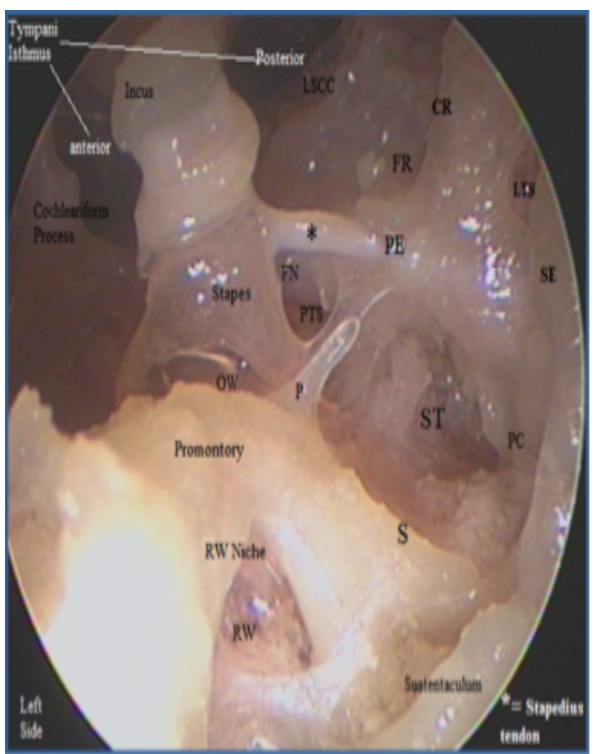

Figure 8. Type 4ST Endoscopic View

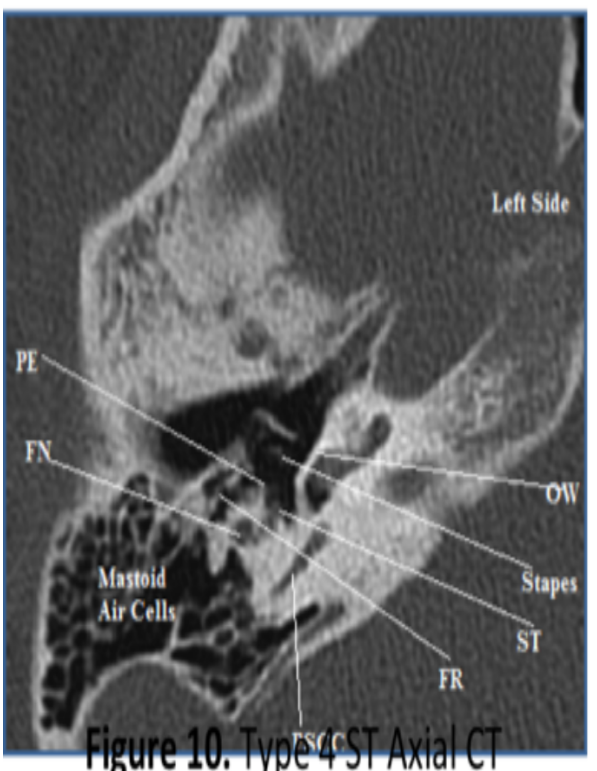

igure 10. Typescst Axialci

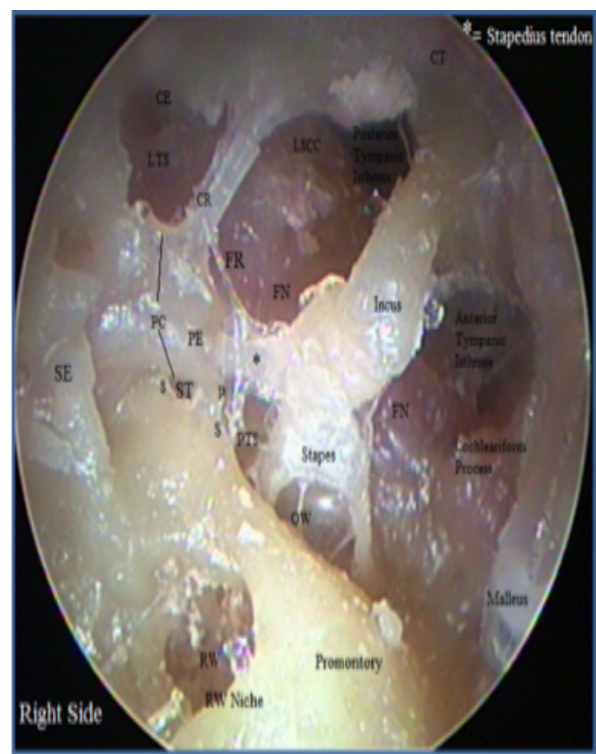

Figure 9. Type 4 ST Endoscopic View

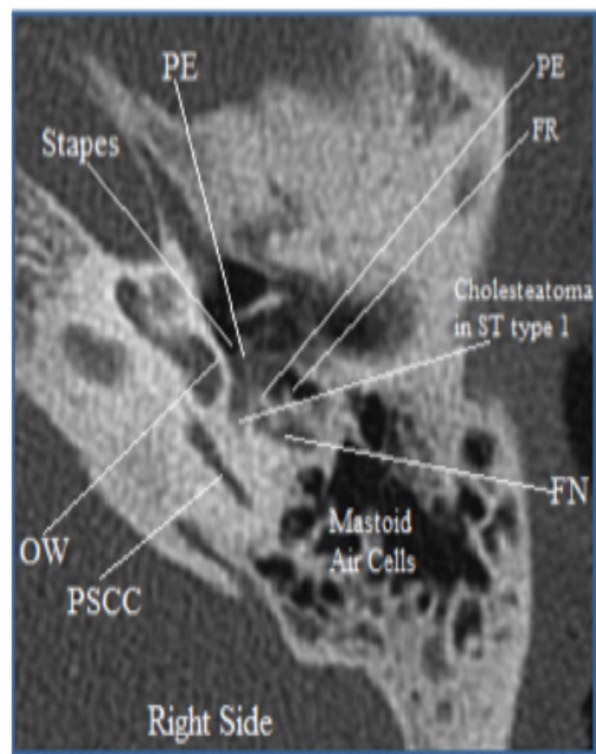

Axial CT

MANSOURA MEDICAL JOURNAL 


\section{DISCUSSION}

The evolution of the ST classification started in 1889 when Steinbrugge $^{3}$ found this sinus could extend beneath the facial nerve. Following studies based the anatomic variations of ST and trials of classifications on single factor either the variations in the size, extension and depth4,5,6,7 or shape 8 . The present work based its proposed ST classification on its development theory mainly combined with its size, extension and depth. In addition, when this study correlated between ST types in CT scans and TBs and at the same time correlated between ST types and possible surgical approaches it provided a clinical significant for the classification and provided a preoperative way to plan ST approach.

\section{CONCLUSION}

ST shape and extension both depend mainly on the extent of its pneumatization, which in turn influences its relations to the surrounding structures in the retrotympanic area. Extensively or unusually pneumatized types need special or combined approaches

Vol. 44, No. 1 \& 2 Jan. \& April, 2015

\section{REFERENCES}

1. Proctor B. Surgical anatomy of the posterior tympanum. (1969) : Ann Otol Rhinol Laryngol; 78:1026-40.

2. Chen B, Yin S, Shen P. (2005) : The Feasibility of the Retrofacial Approach to the Pediatric Sinus Tympani. Otolaryngol Head Neck Surg 2005;133:780-5.

3. Steinbrugge H. (1889) : On sinus tympani. Arch Otolaryngol; 8:53-7.

4. Danaldson JA, Anson BJ, Warpeha RL, et al. (1970) : The surgical anatomy of the sinus tympani. Arch Otolaryngol Head Neck Surg; 91:219-27.

5. Saito R, Igarashi M, Alford BR, et al. (1971) : Anatomical measurement of the sinus tympani. Arch Otolaryngol Head Neck Surg; 94:418-25.

6. Marchioni D, Mattioli F, Alicandri-Ciufelli M, et al. (2009) : Transcanal endoscopic 
Alaa A. Abou-Bieh, MD, Thomas J Haberkamp MD

approach to the sinus tympani: a clinical report. Otol Neurotol

2009;30 (6):758-65.

\section{Pickett BP, Cail WS, Lambert}

PR. (1995) : Sinus tympani: anatomic considerations, computed tomography, and a discussion of the retro-facial approach for removal of disease. Am J Otol; 16:741-50.

8. Marchioni D, Alicandri-Ciufelli $M$, Piccinini $A$, et al. (2010) : Inferior retrotympanum revisited: an endoscopic anatomic study. Laryngoscope;

120

(9):1880-6.

9. Ozturan O, Bauer C, Miller C, et al. (1996) : Dimensions of the sinus tympani and its surgical access via a retrofacial approach. Ann Otol Rhinol Laryngol:
105:776-83.

10. Jasen C. (1968) : The combined approach for tympanoplasty. J Laryngol Otol; 82:779-93.

11. MeKennan KX. (1993) : Endoscopic 'second look' mastoidoscopy to rule out residual epitympnic/mastoid cholesteatoma. Laryngoscope; 103:810-4.

12. Yung MW. (2001): The use of middle ear endoscopy: has residual cholesteatoma been eliminated? J Laryngol Otol; 15:958-61.

13. Marchioni D, Valerini S, Mattioli F, et al. (2015) : Radiological assessment of the sinus tympani: temporal bone HRCT analyses and surgically related findings. Surg Radiol Anat.; 37(4): 385-392. 